\title{
Astragaloside IV inhibits metastasis in hepatoma cells through the suppression of epithelial-mesenchymal transition

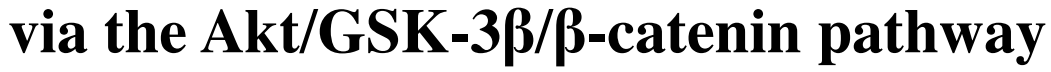

\author{
CHENG-DONG QIN $^{1 *}$, DE-NING MA ${ }^{2 *}$, ZHENG-GANG REN $^{1 *}$, XIAO-DONG ZHU ${ }^{1}$, CHENG-HAO WANG $^{1}$, \\ YING-CONG WANG ${ }^{1}$, BO-GEN YE ${ }^{3}$, MAN-QING CAO ${ }^{1}$, DONG-MEI GAO ${ }^{1}$ and ZHAO-YOU TANG ${ }^{1 *}$ \\ ${ }^{1}$ Liver Cancer Institute, Zhongshan Hospital, Fudan University, Key Laboratory of Carcinogenesis and \\ Cancer Invasion (Fudan University), Chinese Ministry of Education, Shanghai 200032; \\ ${ }^{2}$ Department of Liver Surgery, Fudan University Shanghai Cancer Center, Cancer Hospital, Shanghai 200032; \\ ${ }^{3}$ Department of General Surgery, Changhai Hospital, Second Military Medical University, \\ Shanghai, 200032, P.R. China
}

Received August 7, 2016; Accepted November 4, 2016

DOI: $10.3892 / o r .2017 .5389$

\begin{abstract}
Our previous studies demonstrated that traditional Chinese herbal medicine 'Songyou Yin' inhibited the growth and invasion of hepatocellular carcinoma (HCC) cells, and altered epithelial-mesenchymal transition (EMT) markers in oxaliplatin-treated HCC tissues and cell lines. In the present study, we aimed to explore whether astragaloside IV (AS-IV), a component of 'Songyou Yin', can affect the growth and invasion of HCC cells and the underlying mechanism involved. Human HCC cell lines Huh7 and MHCC97-H, with low and high metastatic potential, respectively, were treated with increasing doses of AS-IV. The Cell Counting Kit-8 (CCK-8), plate clone formation, Transwell, wound healing and immunofluorescence assays were used to investigate the effects of AS-IV on HCC cell proliferation, migration and invasion. The protein expression levels were analyzed by western blotting and immunofluorescence assay. The CCK-8 and plate clone formation assays showed that AS-IV had little effect on the proliferation of HCC cells in vitro. However, the Transwell and wound healing assays demonstrated that AS-IV inhibited the migration and invasion of HCC cells in a dose-dependent manner and the morphology of HCC cells was altered from spindle into oval shaped in the AS-IV pretreated groups. The upregulation of E-cadherin and downregulation of $\mathrm{N}$-cadherin, vimentin, $\alpha$-SMA and Slug were also observed in the AS-IV pretreated groups. Additionally, AS-IV treatment resulted in
\end{abstract}

Correspondence to: Professor Zhao-You Tang, Liver Cancer Institute, Zhongshan Hospital, Fudan University, 180 Feng Lin Road, Shanghai, 200032, P.R. China

E-mail: zytang88@163.com

${ }^{*}$ Contributed equally

Key words: astragaloside IV, hepatocellular carcinoma, epithelialmesenchymal transition a profound decrease in the phosphorylated forms of Akt and GSK-3 $\beta$, which in turn inhibited the expression of $\beta$-catenin. Thus, we conclude that AS-IV attenuates the invasive and migratory abilities of HCC cells through the inhibition of EMT by targeting the Akt/GSK-3 $\beta / \beta$-catenin pathway.

\section{Introduction}

Hepatocellular carcinoma (HCC) is the fifth most prevalent cancer among men and the ninth most common cancer among women worldwide (1) and it is the third leading cause of cancer-related deaths in China (2). Surgical resection is regarded as the primary therapy for early-stage HCC with a 5-year survival rate of $\sim 60-70 \%$ in patients who have undergone surgical treatment $(3,4)$. However, postoperative recurrence is very common (5) and intrahepatic metastases, vascular invasion and satellite foci are the main reasons for this recurrence (4). Therefore, it is urgent to understand the mechanisms involved in HCC metastasis. Activation of the epithelial-mesenchymal transition (EMT) process is considered to be a critical event for the metastasis of tumors, particularly in the early stage (6). After EMT, HCC cells lose their epithelial phenotype with the downregulation of E-cadherin and transform to a mesenchymal phenotype with the upregulation of multiple mesenchymal genes (N-cadherin, vimentin and $\alpha$-SMA) (6).

Chinese herbal medicine has been demonstrated to have a therapeutic effect on tumors $(7,8)$. The Chinese herbal medicine 'Songyou Yin' (SYY) contains 5 Chinese medicinal herbal extracts with chromatographic fingerprints in the following ratios (w/w): Astragalus membranaceus Bge, 14.3\%; Salvia miltiorrhiza Bge, 14.3\%; Lycium barbarum L., 23.8\%; Crataegus pinnatifida Bge, $23.8 \%$ and Trionyx sinensis Wiegmann, 23.8\% (all from China) (9). In our previous studies, we found that SYY suppressed the growth and invasion of HCC cells (9). Furthermore, SYY also enhanced the sensitivity of HCC cells to chemotherapy through the inhibition of the stemness of the hepatoma cells (10). Astragalus membranaceus is 
a traditional Chinese herb. It is usually used to treat edemas of acute nephritis, colds, vulnus and many other diseases (11). Various recent studies demonstrated that Astragalus membranaceus has also anticancer bioactivity (12-14). Due to its complex composition and the lack of scientific basis to support its effects, it may be useful to investigate which of its components has an antitumor effect. Astragaloside-IV (AS-IV; chemical name, 3-O- $\beta$-D-xylopyranosyl-6- $O-\beta-\mathrm{D}$ glucopyranosyl-cycloastragenol) is a primary bioactive constituent of Astragalus membranaceus (15), which has an explicit chemical formula and an exact molecular weight $(16,17)$ (Fig. 1A). It was reported that AS-IV enhanced the expression of smad7 to suppress the TGF- $\beta 1$ induced EMT of peritoneal mesothelial cells (18). Additionally, various studies also proved that AS-IV was able to decrease the production of reactive oxygen species (ROS), thus, glycated albumin induced EMT which was inhibited in renal tubular cells (19). Our previous study also showed that SYY functioned in changes in EMT-related genes in oxaliplatin-treated HCC tissues and cell lines (20). In the present study, the aim was to investigate the effects of AS-IV on the proliferation, invasion and migration of HCC cells and the underlying mechanisms involved.

\section{Materials and methods}

Cell line culture. The human HCC cell line Huh7 with low metastatic potential was obtained from the Cell Bank of the Chinese Academy of Sciences (Shanghai, China), and the human HCC cell line MHCC97-H with high metastatic potential was established at the authors' institution (Liver Cancer Institute, Fudan University, Shanghai, China) (21). All the HCC cell lines were cultured in Dulbecco's modified Eagle's medium (DMEM; Invitrogen, Carlsbad, CA, USA) containing $10 \%$ fetal bovine serum (FBS) and $100 \mathrm{U} / \mathrm{ml}$ penicillin and $50 \mathrm{mg} / \mathrm{ml}$ streptomycin. All cells were incubated at $37^{\circ} \mathrm{C}$ with a humidified atmosphere of $5 \% \mathrm{CO}_{2}$.

Reagents and antibodies. In the in vitro study, an AS-IV monomer (purchased from the National Institutes for Food and Drug Control, Beijing, China), a lyophilized powder with a purity of $99.99 \%$, first dissolved in dimethyl sulfoxide (DMSO) and then diluted with phosphate-buffered saline (PBS) to the required concentration, was used in the assays. GSK690693 (Selleck Chemicals, Houston, TX, USA), an Akt-specific inhibitor was first dissolved in DMSO, and then added to the cell culture medium for the required concentration. Antibodies used for immunofluorescence and immunoblotting were as follows: rabbit anti-human monoclonal E-cadherin, rabbit anti-human monoclonal $\mathrm{N}$-cadherin, rabbit anti-human monoclonal vimentin, rabbit anti-human monoclonal Slug (all from Cell Signaling Technology, Beverly, MA, USA), rabbit anti-human monoclonal $\alpha$-SMA (Abcam, Cambridge, UK), rabbit anti-human monoclonal $\mathrm{P}-\mathrm{AKT}$ and rabbit anti-human monoclonal AKT (both from Cell Signaling Technology), rabbit anti-human monoclonal $\beta$-catenin (Abcam), mouse anti-human monoclonal GAPDH and mouse anti-human monoclonal $\beta$-tubulin (both from Beyotime, Haimen, China).

Cell proliferation assays. Huh7 and MHCC97-H cells were incubated with different concentrations of AS-IV for $72 \mathrm{~h}$, and then plated in 96-well plates and exposed to increasing doses of AS-IV for 24, 48 and $72 \mathrm{~h}$. Cell proliferation assays were performed by the Cell Counting Kit-8 (CCK-8; Dojindo Laboratories, Kumamoto, Japan). The optical density was read at a wavelength of $450 \mathrm{~nm}$.

For colony formation assays, we counted one thousand cells which were added to 6-well plates (Corning Inc., Corning, NY, USA), and cultured with 1\% FBS DMEM with various concentration of AS-IV. The medium was changed every 4 days. After 14 days, ice-cold $4 \%$ paraformaldehyde was used to fix the colonies. The colonies were stained with crystal violet and the images were captured using a camera.

Cell migration and invasion assays. Quantitative cell migration and invasion were assessed using Transwell assays (Boyden chambers; Corning Inc.). First, Huh7 and MHCC97-H cells were pretreated with different doses of AS-IV for $72 \mathrm{~h}$. Due to their different biological characteristics, such as morphology and mobility, $\sim 5 \times 10^{4} \mathrm{Huh} 7$ cells/well and $3 \times 10^{4} \mathrm{MHCC} 97-\mathrm{H}$ cells/well in DMEM, respectively, were added into the upper chamber of each well of 24-well plates containing $8.0-\mu \mathrm{m}$ pore size membranes, and the lower chamber was filled with DMEM containing 5\% FBS. Cells were allowed to migrate for $24 \mathrm{~h}$ at $37^{\circ} \mathrm{C}$. The crystal violet was used on stained cells that had reached the underside of the membrane. Finally, 5 random fields were photographed at a magnification of x100, and the number of stained cells was counted. Transwell assays to assess the invasion were similarly performed except that $90 \mu \mathrm{l}$ diluted Matrigel (1:9 DMEM; BD Biosciences, San Jose, CA, USA) was added into each well $3 \mathrm{~h}$ before cells were seeded in the upper chamber.

The wound healing assay was also used to evaluate cell migration. Briefly, $\sim 5 \times 10^{5} \mathrm{HCC}$ cells/well were added into 6 -well plates. Cells were incubated overnight to produce a confluent monolayer. A $10-\mu 1$ pipette tip was used to make a straight scratch on the monolayer of the cells attached to the bottom of a Petri dish. The suspended cells were washed 3 times with PBS. Then, FBS-free DMEM with various concentrations of AS-IV were added to each well for $24 \mathrm{~h}$. The wound area was photographed under an inverted microscope at 0 and $24 \mathrm{~h}$.

Immunofluorescence assays. HCC cells were first pretreated with increasing doses of AS-IV. Then, $\sim 4 \times 10^{4}$ cells/well were added into a glass bottom dish. After cells attached to the bottom, 4\% paraformaldehyde was used to fix the cells for $15 \mathrm{~min}$ at room temperature. Then, cells were treated with $0.2 \%$ Triton X-100 for $15 \mathrm{~min}$ with the purpose of permeabilization and incubated in a blocking buffer (5\% BSA in PBS, pH 7.4) at room temperature for $30 \mathrm{~min}$. Subsequently, the cells were incubated with the respective primary antibody overnight at $4^{\circ} \mathrm{C}$. The next day, the cells were incubated with the corresponding FITC-conjugated secondary antibody (Beyotime) in the dark for $60 \mathrm{~min}$ at room temperature, and DAPI solution was added in the last 10 min during this process. The cells were observed and photographed under a fluorescence microscope.

Western blotting. Cells were lysed using ice-cold RIPA buffer (150 mM NaCl, 50 mM Tris- $\mathrm{HCl}, \mathrm{pH} 8.0,0.1 \%$ SDS, $1 \%$ Triton X-100) containing protease and phosphatase inhibitors. 
A

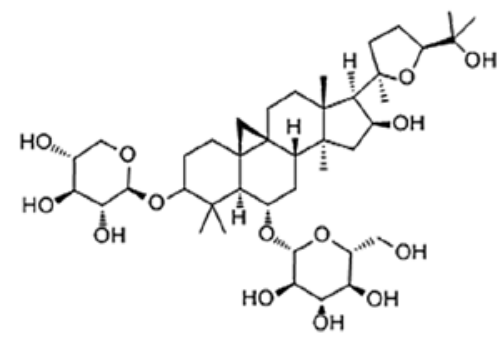

B
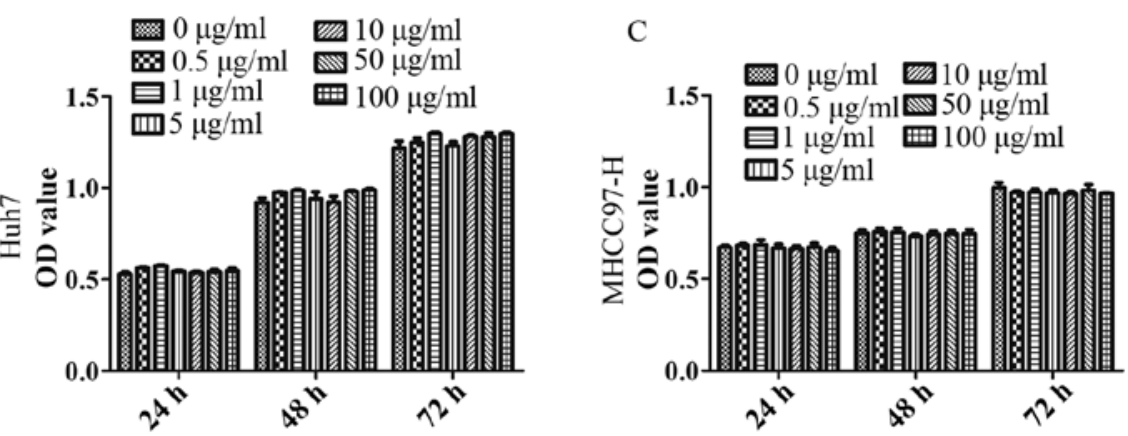

D

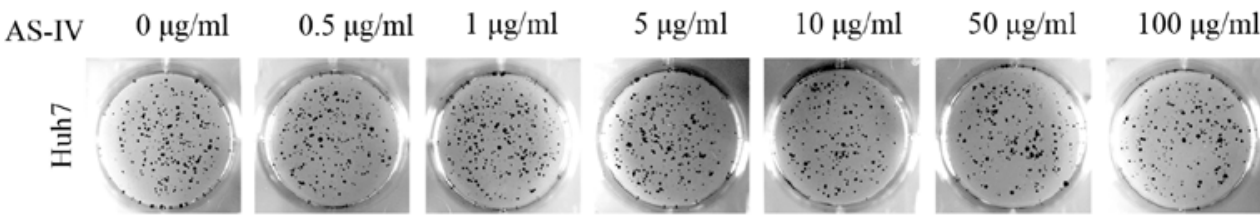

E
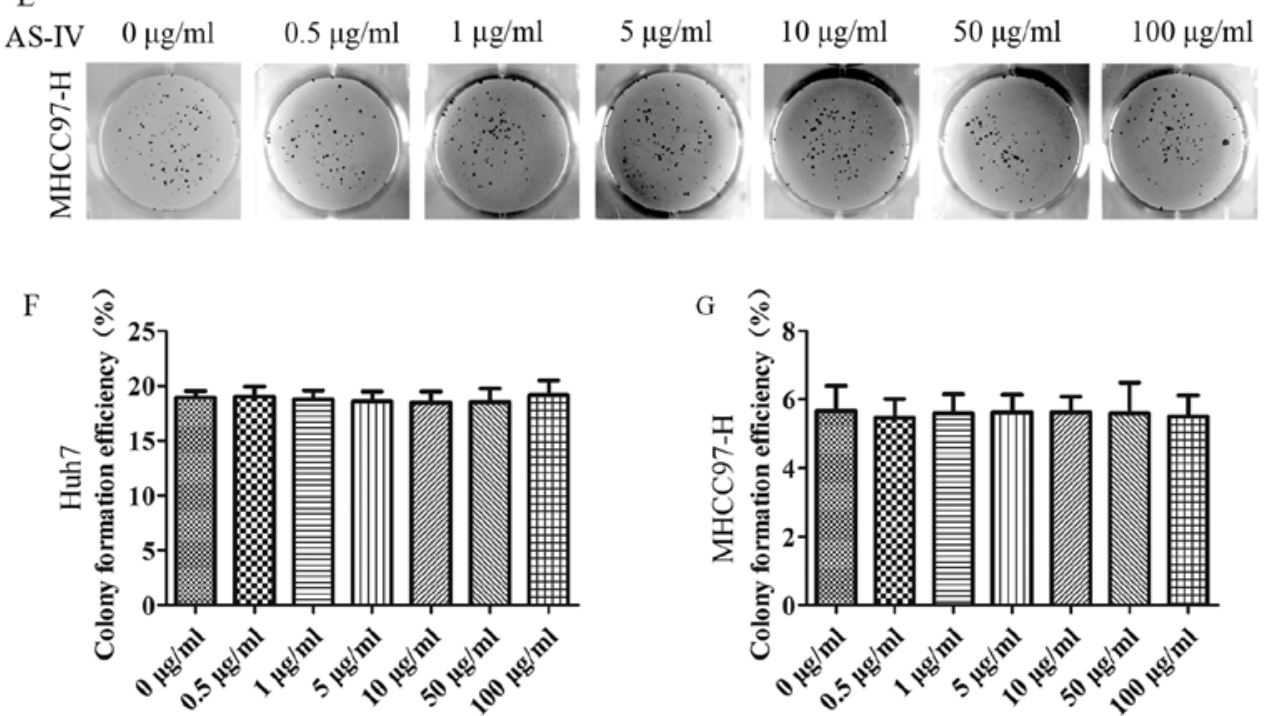

Figure 1. Astragaloside IV (AS-IV) has no effect on the proliferation of HCC cells in vitro. (A) The chemical structure of AS-IV. (B and C) Huh7 and MHCC97-H cells were incubated with increasing doses of AS-IV $(0,0.5,1,5,10,50$ and $100 \mu \mathrm{g} / \mathrm{ml})$ for $72 \mathrm{~h}$, and a CCK-8 assay was used to measure the proliferation of HCC cells. The results revealed that there were no significant differences between each of the groups at any of the time-points. (D and E) The plate clone formation assay was also used to evaluate the effects of AS-IV on the proliferation of HCC cells. The results demonstrated that there was no significant difference between the groups with different AS-IV concentrations. ( $F$ and G) The colony formation efficiency was counted and compared in the diagrams.

Equal amounts of proteins from each group were subjected to SDS-PAGE gel and transferred to polyvinylidene difluoride (PVDF) membranes. The membranes were blocked with 5\% fat-free milk for $1 \mathrm{~h}$, and then incubated with the respective primary antibody overnight at $4^{\circ} \mathrm{C}$. Afterwards, the membranes were washed in Tris-buffered saline with Tween-20 (TBST) 3 times and incubated with the corresponding HRP-conjugated secondary antibody for $1 \mathrm{~h}$ at room temperature. Blots were visualized with an enhanced chemiluminescence (ECL) detection kit (Millipore, Bedford, MA, USA) and analyzed using Quantity One 1-D Analysis Software (Bio-Rad, San Francisco, CA, USA).

Statistical analysis. All the experimental data were analyzed using Excel 2016 for Windows (Microsoft, Redmond, WA, USA) and SPSS 19.0 for Windows (SPSS, Inc., Chicago, 
A
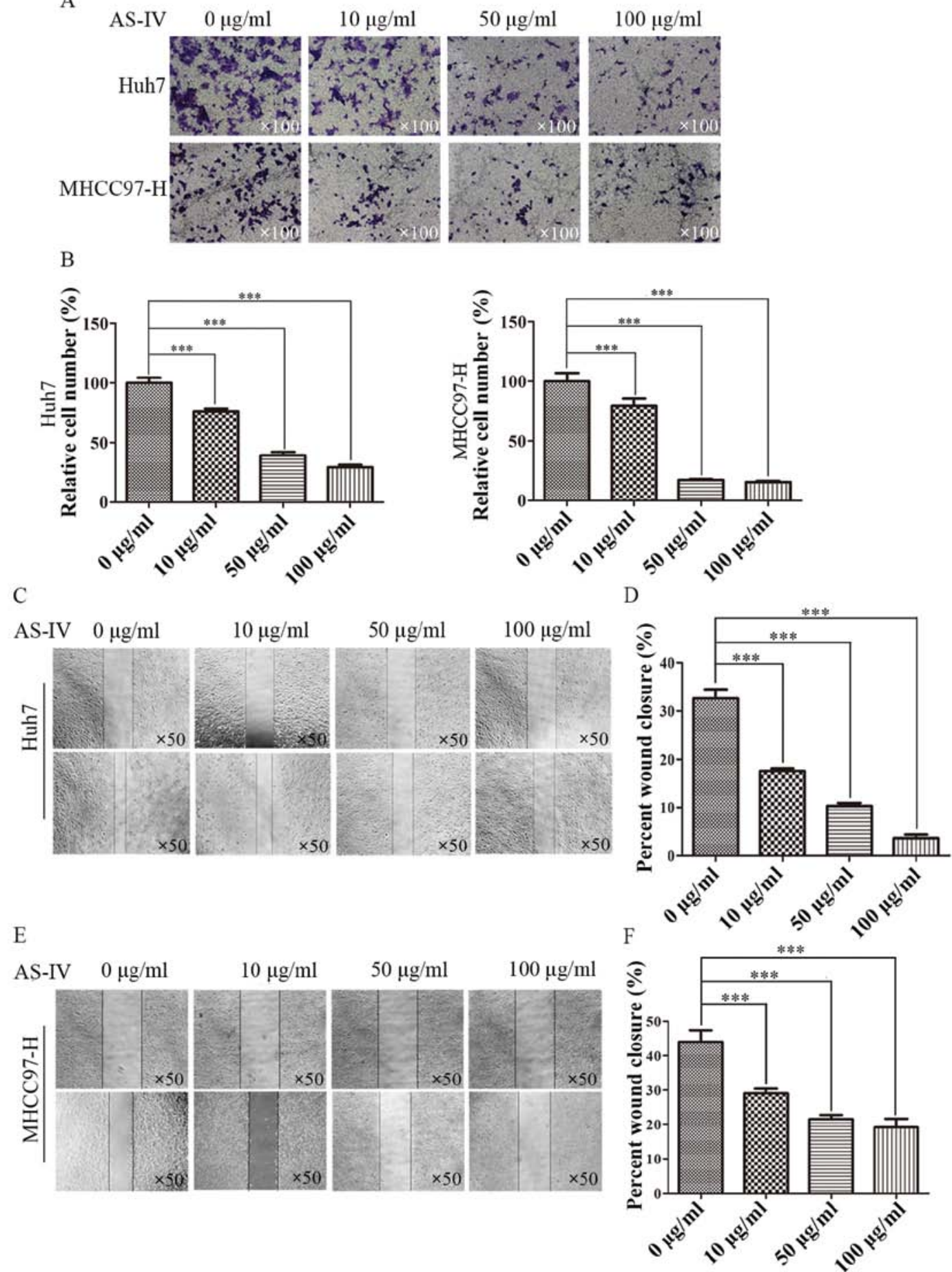

Figure 2. Astragaloside IV (AS-IV) suppresses the migration of Huh7 and MHCC97-H cells in vitro. (A) Transwell assays were performed to evaluate the effects of AS-IV on the metastasis of both cell lines. The results revealed that AS-IV $(5,10,50$ and $100 \mu \mathrm{g} / \mathrm{ml})$ significantly inhibited the ability of cells to pass through the membrane compared with the control group $(0 \mu \mathrm{g} / \mathrm{ml})$ in both cell lines. (B) The number of cells that passed through the membrane were counted and compared in the diagrams; ${ }^{* * * *} \mathrm{p}<0.001$. (C and $\mathrm{E}$ ) The results of the wound healing assays also revealed that AS-IV inhibited the migration of Huh7 and MHCC97-H cells. (D and F) The percent of wound closure was assessed and compared in the diagrams; ${ }^{* * *} \mathrm{p}<0.001$.

IL, USA). An unpaired Student's t-test was used to compare quantitative variables and a level of $\mathrm{p}<0.05$ was considered to indicate a statistically significant result.

\section{Results}

AS-IV suppresses HCC cell migration and invasion, but not proliferation. A series of increasing doses $(0-100 \mu \mathrm{g} / \mathrm{ml})$ of
AS-IV was used to treat MHCC97-H and Huh7 cells in the CCK-8 and plate clone formation assays, in order to evaluate the effects of AS-IV on MHCC97-H and Huh7 cell proliferation. In the CCK-8 assay, there were no significant differences in cell proliferation or apoptosis between each of the groups at any of the time-points, as shown in Fig. $1 \mathrm{~B}$ and $\mathrm{C}$ at $72 \mathrm{~h}$. In addition, in the plate clone formation assay (2 weeks), the results demonstrated that the average clone formation rate was 

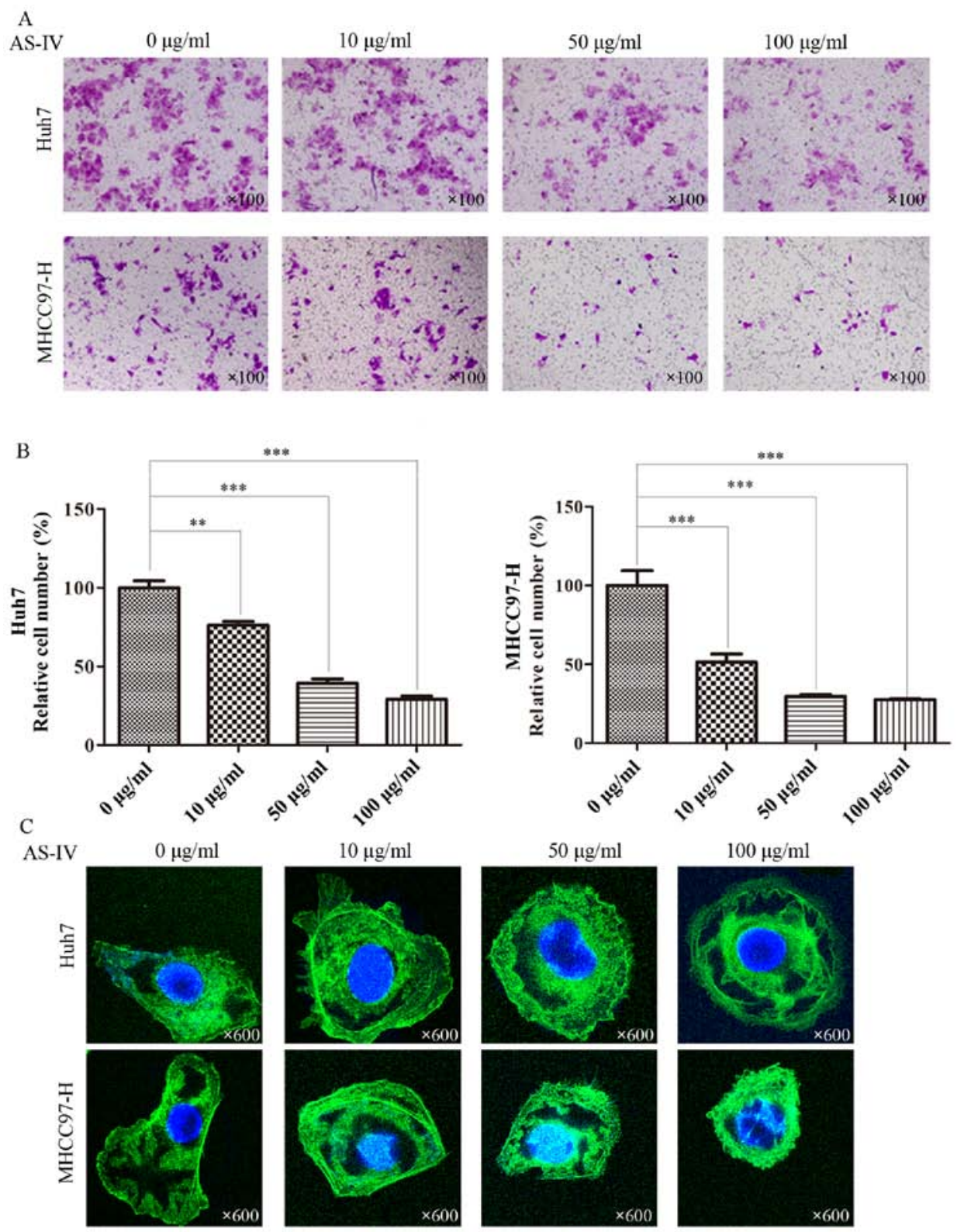

Figure 3. Astragaloside IV (AS-IV) suppresses the invasion of Huh7 and MHCC97-H cells in vitro. (A) The results of the Transwell assays with Matrigel demonstrated that AS-IV (5, 10, 50 and $100 \mu \mathrm{g} / \mathrm{ml})$ suppressed the invasion of HCC cells. (B) The number of cells that passed through the membrane was counted and are compared in the graphs; ${ }^{* *} \mathrm{p}<0.01 ;{ }^{* * *} \mathrm{p}<0.001$. (C) The cellular morphology and F-actin fibers of Huh7 and MHCC $97-\mathrm{H}$ cells were altered after incubation with AS-IV.

not significantly different between each group (Fig. 1D-F). Consequently, we chose 10,50 and $100 \mu \mathrm{g} / \mathrm{ml}$ of AS-IV for further experiments.

In order to evaluate the effects of AS-IV on HCC cell migration and invasion, Transwell assays were performed. After $24 \mathrm{~h}$, we stained the cells that had passed through the chamber membrane and counted the number of cells from each group. Compared with the control group, the number of migrating cells pretreated with AS-IV decreased with increasing AS-IV concentration. The relative percentage of migratory Huh7 cells treated with 10,50 and $100 \mu \mathrm{g} / \mathrm{ml} \mathrm{AS}-\mathrm{IV}$ was $57.6 \pm 3.4,33.5 \pm 2.2$ and $20.7 \pm 1.8 \%$ compared to the control group, respectively (Fig. 2A and B). The relative percentage of migratory MHCC $97-\mathrm{H}$ cells treated with 10,50 and $100 \mu \mathrm{g} / \mathrm{ml}$ AS-IV was $51.3 \pm 5.2,29.7 \pm 1.1$ and $27.7 \pm 0.5 \%$ of control group, respectively (Fig. 2A and B).
In the wound healing assay, we found that AS-IV significantly suppressed the wound healing in the experiments with both Huh7 and MHCC97-H cells. The closure rates of Huh7 cells treated with $0,10,50$ and $100 \mu \mathrm{g} / \mathrm{ml}$ AS-IV were $32.6 \pm 1.9$, 17.5 $\pm 0.6,10.3 \pm 0.6$ and $3.7 \pm 0.7 \%$, respectively (Fig. $2 \mathrm{C}$ and D). The closure rates of MHCC97-H cells treated with $0,10,50$ and $100 \mu \mathrm{g} / \mathrm{ml}$ AS-IV were 44.1 $\pm 3.4,29.1 \pm 1.3,21.5 \pm 1.2$ and $19.2 \pm 2.5 \%$, respectively (Fig. $2 \mathrm{E}$ and F).

Transwell assays with Matrigel further revealed that AS-IV clearly restrained the invasive capacity of Huh7 and MHCC97-H cells as compared with the control group. In the group of Huh7 cells treated with 10,50 and $100 \mu \mathrm{g} / \mathrm{ml}$ of AS-IV, the relative percentage of migratory cells was $76.2 \pm 2.3,39.3 \pm 2.8$ and $29.1 \pm 2.0 \%$ compared to the control group (Fig. 3A and B). In the group of MHCC97-H cells treated with 10, 50 and $100 \mu \mathrm{g} / \mathrm{ml}$ of AS-IV, the relative percentage of migratory cells 

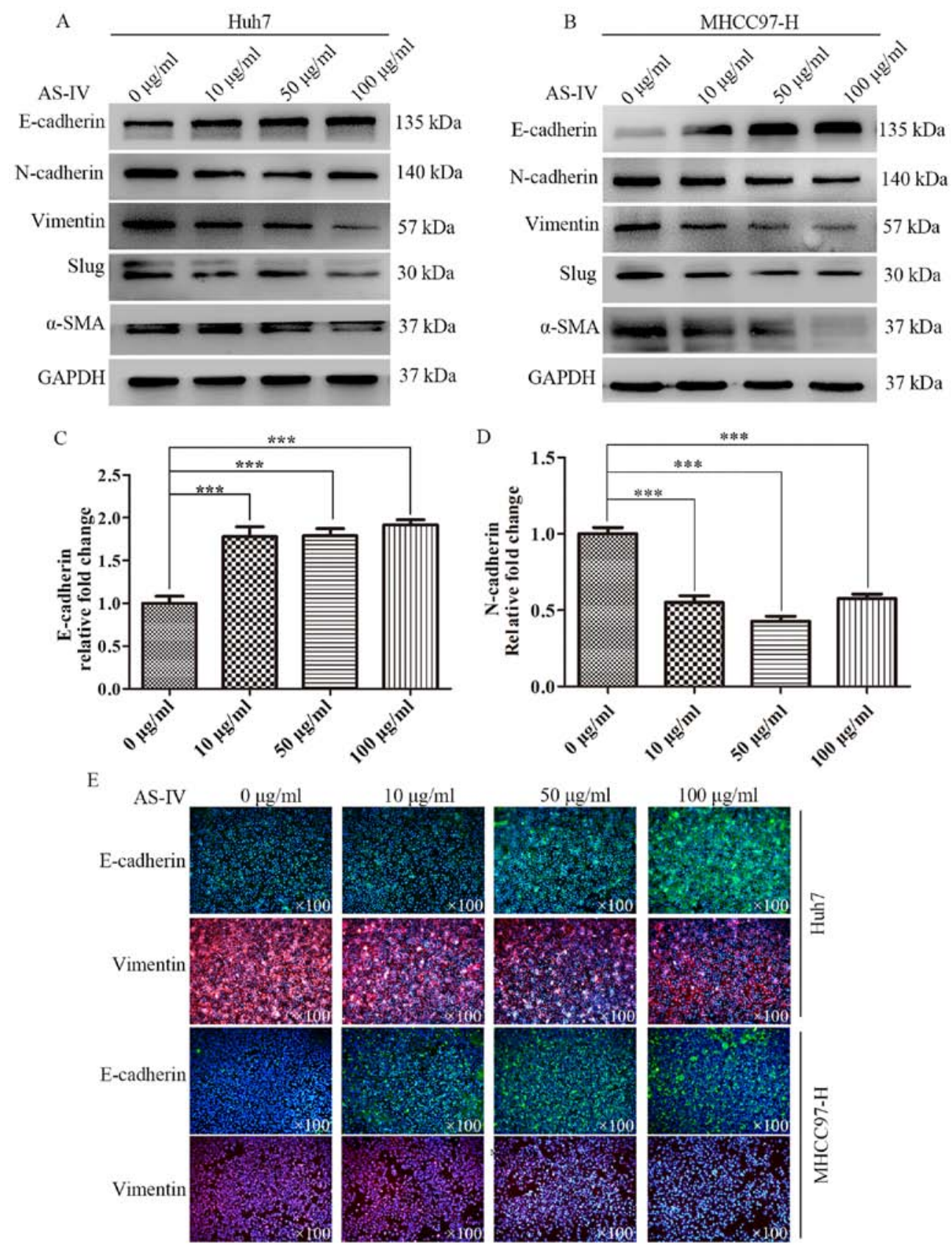

Figure 4. Astragaloside IV (AS-IV) inhibits EMT in hepatoma cells in vitro. (A and B) Western blotting revealed that the changes in proteins associated with EMT were consistent with both Huh7 and MHCC97-H cell lines, after pretreatment with AS-IV (e.g., upregulation of E-cadherin and downregulation of N-cadherin, vimentin, $\alpha$-SMA and Slug); GAPDH was used as an internal control. (C and D) The gray-scale values of the protein bands of E-cadherin and $\mathrm{N}$-cadherin of Huh7 cells were assessed and are compared in the diagrams; ${ }^{* * *} \mathrm{p}<0.001$. (E) The immunofluorescent assays were used to assess the effects of AS-IV on E-cadherin and vimentin expression. The results showed that AS-IV $(5,10,50$ and $100 \mu \mathrm{g} / \mathrm{ml})$ markedly increased the fluorescence intensity of E-cadherin and decreased the fluorescence intensity of vimentin in both Huh7 and MHCC97-H cell lines.

was $79.5 \pm 6.0,17.8 \pm 0.8$ and $16.2 \pm 1.0 \%$ compared to the control group (Fig. 3A and B).

In order to further demonstrate these results, we studied AS-IV-induced cytoskeletal and morphological changes in Huh7 and MHCC97-H cells. The Huh7 and MHCC97-H cells with spindle-shaped cellular morphology and stretched F-actin fibers were altered to a cobblestone appearance and shrinkable F-actin fibers after incubation with AS-IV (Fig. 3C).

AS-IV suppresses EMT in HCC cells. The aforementioned results demonstrated that after AS-IV treatment, the morphology of HCC cells changed from a spindle into an oval shaped. In addition, the mobility and invasion of HCC cells were inhibited compared with the control group. We speculated that AS-IV inhibits EMT in HCC cells. Western blotting was used to further examine the effects of AS-IV on epithelial marker (E-cadherin) and mesenchymal markers (N-cadherin, vimentin, Slug and $\alpha$-SMA). After incubating Huh7 cells with 10,50 and $100 \mu \mathrm{g} / \mathrm{ml} \mathrm{AS-IV} \mathrm{for} 48 \mathrm{~h}$, the expression level of E-cadherin significantly increased to $1.77 \pm 0.10-$, $1.78 \pm 0.07$ - and 1.92 \pm 0.04 -fold compared with the control group (Fig. 4A and C). Conversely, mesenchymal markers markedly decreased. The expression level of $\mathrm{N}$-cadherin decreased to $0.55 \pm 0.04-, 0.43 \pm 0.03$ - and $0.57 \pm 0.02$-fold compared 


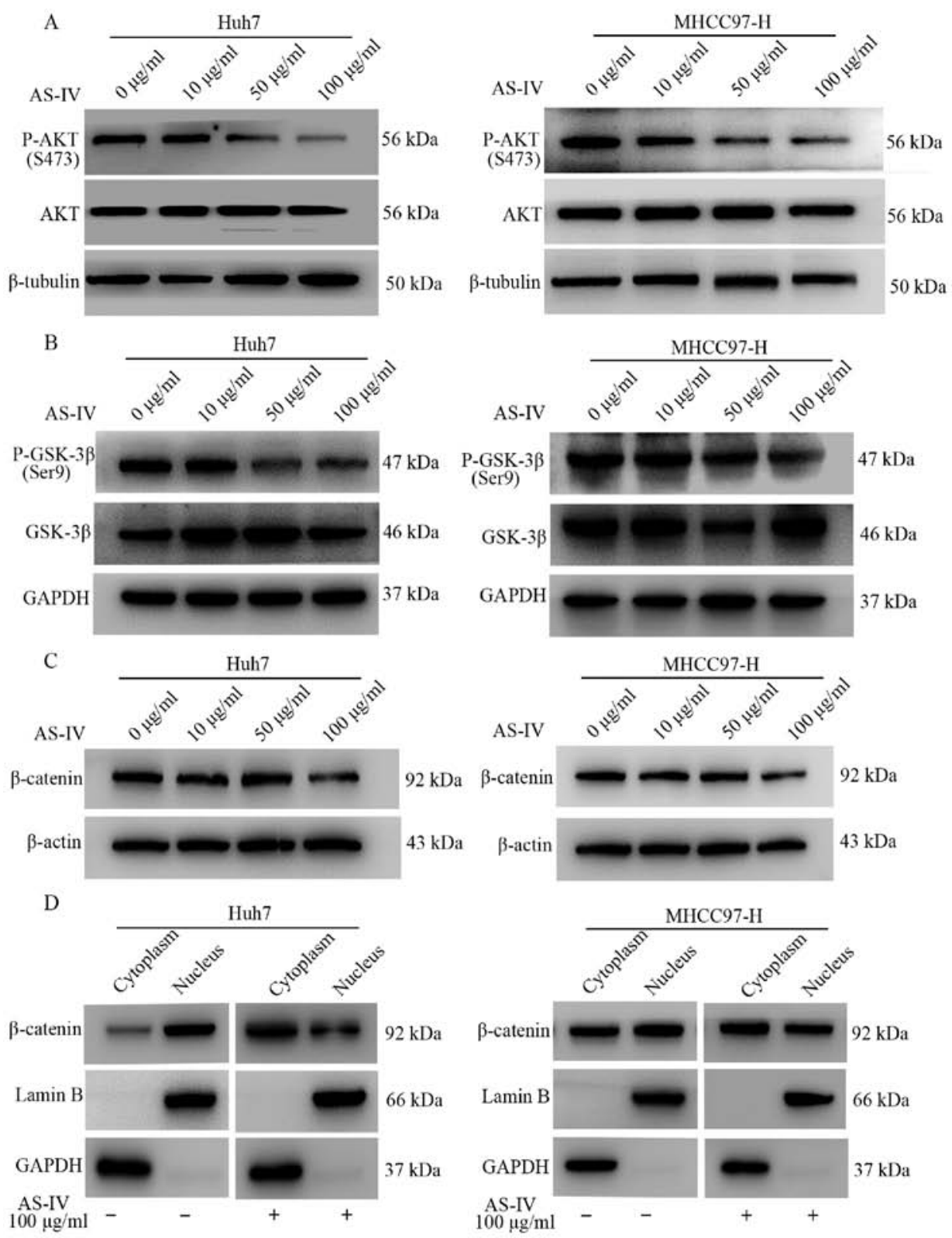

Figure 5. Astragaloside IV (AS-IV) suppresses the EMT process by targeting the Akt/GSK-3 $\beta / \beta$-catenin pathway. (A) The protein expression levels were detected by western blotting. The results showed that the phosphorylation level of Akt (P-AKT) was suppressed in the AS-IV-treated group (5, 10, 50 and

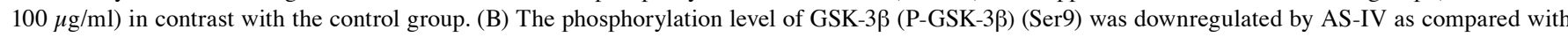
the control group. (C) Western blotting revealed that the expression of $\beta$-catenin was clearly inhibited by AS-IV (100 $\mu \mathrm{g} / \mathrm{ml})$ as compared with the control group. (D) Western blotting revealed that AS-IV $(100 \mu \mathrm{g} / \mathrm{ml})$ decreased the accumulation of $\beta$-catenin in contrast with the control group.

to the control group (Fig. 4A and D). The same phenomena were observed in the MHCC97-H cells treated with AS-IV; the increased expression of E-cadherin was accompanied by decreased expression of mesenchymal cell markers (Fig. 4B).

In order to further validate the aforementioned results, we performed an immunofluorescence assay to test the influence of AS-IV on E-cadherin and vimentin in both cell lines. After incubating both cell lines with AS-IV for $48 \mathrm{~h}$, we found that AS-IV greatly increased E-cadherin expression and significantly decreased vimentin expression in a concentration-dependent manner (Fig. 4E).

$A S$-IV inhibits EMT in HCC cells through the regulation of the

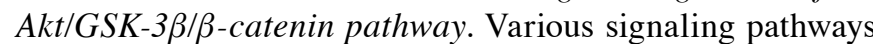
have been proven to play important roles in the EMT process.
It has been confirmed that the oncogenic serine/threonine kinase AKT regulates EMT in many tumors $(22,23)$. AKT suppresses transcription of the E-cadherin gene, resulting in the decrease of E-cadherin protein expression and loose intercellular junctions (24). In order to verify whether AKT could be modulated by AS-IV, western blotting was performed. The results demonstrated that AS-IV attenuated the expression of phosphorylated AKT (P-AKT), particularly at the dose of $100 \mu \mathrm{g} / \mathrm{ml}$ (Fig. 5A).

A previous study showed that phosphorylated Akt suppresses the activity of GSK-3 $\beta$ by phosphorylating its Ser9 residues, which is accompanied by the accumulation of $\beta$-catenin in the nucleus and activation of the Wnt pathway. In the present study, we found that 50 and $100 \mu \mathrm{g} / \mathrm{ml}$ of AS-IV markedly downregulated the phosphorylation level of GSK-3 $\beta$ 
A

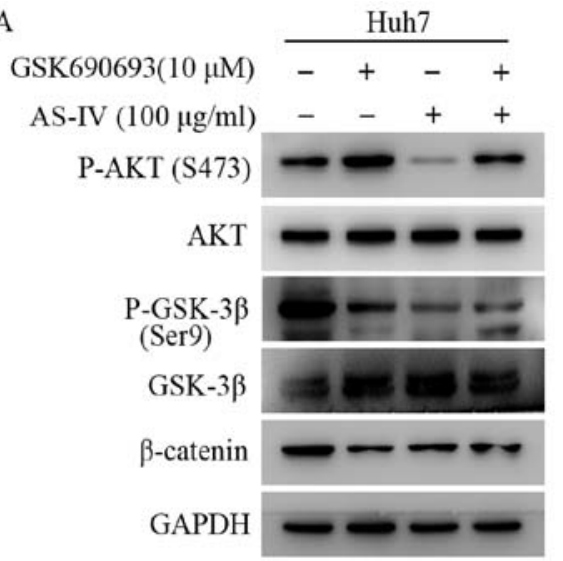

$56 \mathrm{kDa}$

$56 \mathrm{kDa}$

$47 \mathrm{kDa}$

$46 \mathrm{kDa}$

$92 \mathrm{kDa}$

$37 \mathrm{kDa}$

B

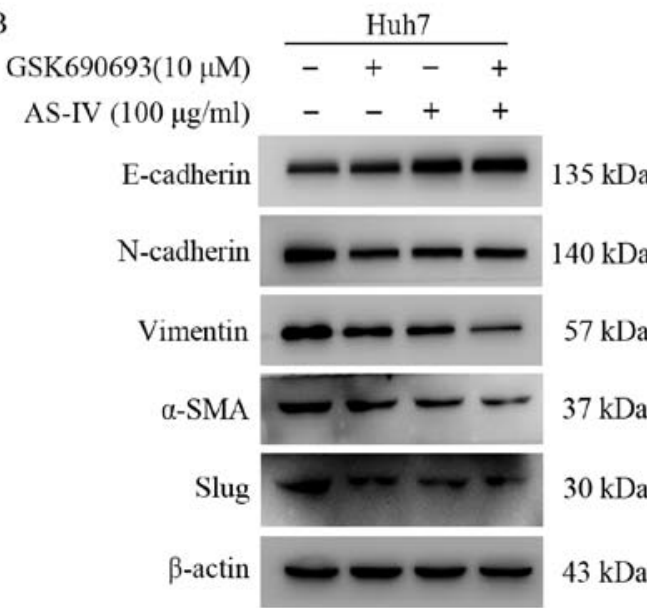

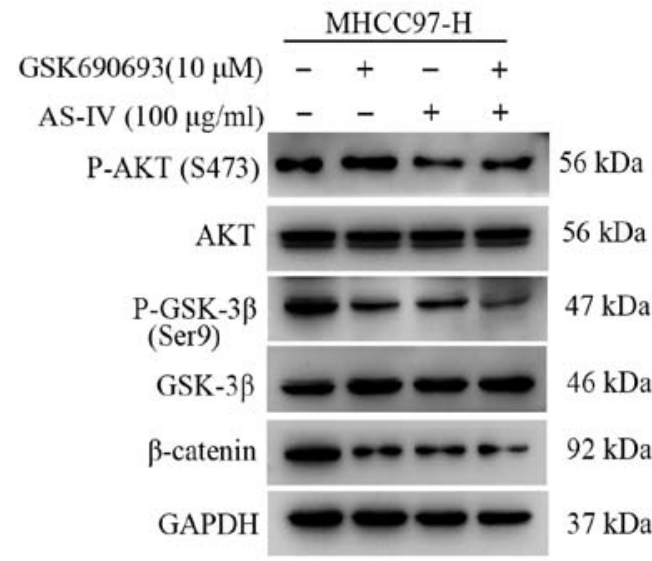

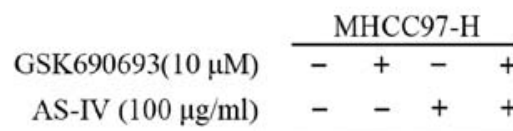

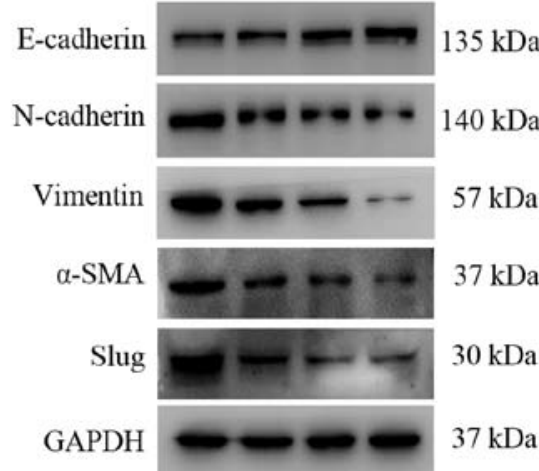

Figure 6. Confirmation of the relationship between astragaloside IV (AS-IV) and the Akt/GSK-3 $\beta / \beta$-catenin pathway using the Akt inhibitor. (A) The results from western blotting demonstrated that the phosphorylation level of Akt (P-AKT) at the S473 site was increased in the GSK690693-treated group (10 $\mu \mathrm{M})$ and could be decreased by combining AS-IV $(100 \mu \mathrm{g} / \mathrm{ml})$ and GSK690693 $(10 \mu \mathrm{M})$. The phosphorylation of GSK-3 $\beta$ (P-GSK-3 $\beta)$ (Ser9) and the expression of $\beta$-catenin were inhibited by AS-IV $(100 \mu \mathrm{g} / \mathrm{ml})$, GSK690693 $(10 \mu \mathrm{M})$ and their combined use. (B) The results from western blotting also revealed that both AS-IV $(100 \mu \mathrm{g} / \mathrm{ml})$ and GSK690693 $(10 \mu \mathrm{M})$ upregulated the expression of E-cadherin and downregulated the expression of N-cadherin, vimentin, $\alpha$-SMA and Slug. Thus, the combination of the two may be more effective.

in both Huh7 and MHCC97-H cells, as compared with the control group (Fig. 5B).

Past studies have suggested that once the expression of E-cadherin is suppressed, the $\beta$-catenin/E-cadherin complex at the cell membrane is also destroyed and a massive amount of $\beta$-catenin enters the nucleus $(25,26)$. Therefore, the influence of AS-IV on the expression of $\beta$-catenin was investigated. The results showed that 10 and $50 \mu \mathrm{g} / \mathrm{ml}$ of AS-IV had little influence on $\beta$-catenin in both cell lines, but the concentration of $100 \mu \mathrm{g} / \mathrm{ml}$ of AS-IV significantly suppressed the expression of the $\beta$-catenin protein (Fig. 5C). Next, we investigated the effects of AS-IV on the location of the $\beta$-catenin protein. The results from western blotting demonstrated that $100 \mu \mathrm{g} / \mathrm{ml}$ of AS-IV inhibited the accumulation of $\beta$-catenin in the nucleus as compared with the control group in both HCC cell lines (Fig. 5D).

In order to further confirm the relationship between AS-IV and the Akt/GSK-3// $\beta$-catenin pathway, GSK690693, an AKT inhibitor, was used to incubate Huh7 and MHCC97-H cells with or without AS-IV for $24 \mathrm{~h}$. The results demonstrated that GSK690693 increased the phosphorylation level of AKT at the Ser473 site in both cell lines (Fig. 6A). This was consistent with the feedback mechanism previously demonstrated $(27,28)$. In addition, treatment with GSK690693 decreased the phosphorylation level of GSK-3 $\beta$ and the expression level of $\beta$-catenin in the Huh7 and MHCC97-H cells (Fig. 6A). GSK690693 also inhibited the occurence of EMT in both cell lines (Fig. 6B). In the group using both the AS-IV and GSK690693, the level of P-AKT at the Ser473 site was decreased, while, the P-GSK-3 $\beta$ level was further decreased in both cell lines (Fig. 6A). In addition, the expression of $\beta$-catenin was also inhibited (Fig. 6A). The results of western blotting also demonstrated that using AS-IV and GSK690693 together increased the epithelial marker (E-cadherin) and decrease the mesenchymal markers (N-cadherin, vimentin, Slug and $\alpha$-SMA) more effectively in the HCC cells (Fig. 6B). Collectively, these results suggest that AS-IV inhibits the EMT of HCC through the modulation of the Akt/GSK-3 $\beta / \beta$-catenin pathway (Fig. 7).

\section{Discussion}

Invasion and metastasis are the main causes of tumor relapse, and are regarded as important characteristics of HCC. EMT plays an important role in the process of tumor invasion and metastasis, particularly in the early stage (6). Traditional Chinese medicine contains a variety of active ingredients. 


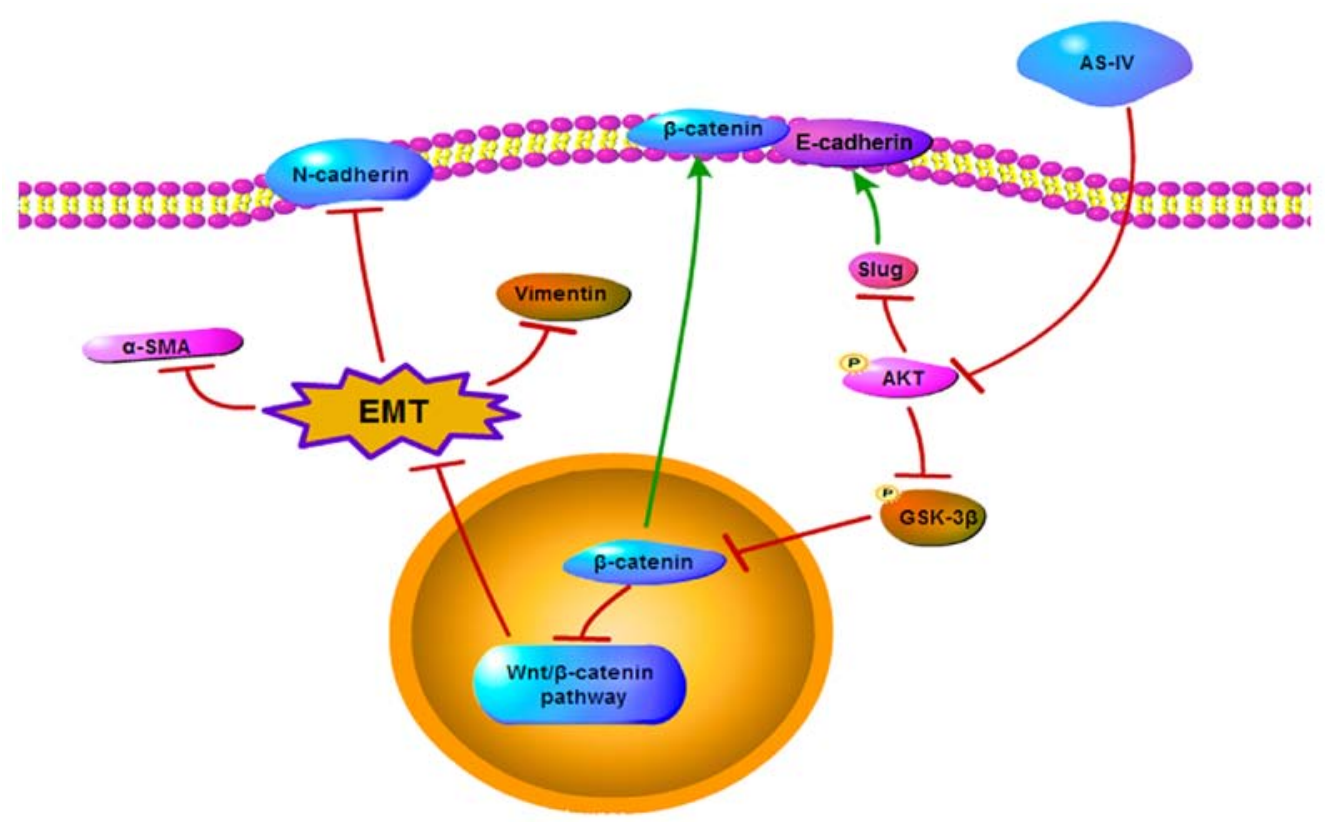

Figure 7. Astragaloside IV (AS-IV) inhibits EMT of HCC through the modulation of the Akt/GSK-3 $\beta / \beta$-catenin pathway. EMT, epithelial-mesenchymal transition; HCC, hepatocellular carcinoma.

Many types of ingredients play a role in the inhibition of tumor metastasis (29). In the present study, we investigated the effects of AS-IV on HCC cell proliferation and metastasis in vitro. Our results demonstrated that AS-IV had little effect on the proliferation of HCC cells in vitro. However, we found that AS-IV inhibited the invasion and metastasis of HCC cells in a concentration-dependent manner in vitro. Moreover, AS-IV significantly enhanced the expression of E-cadherin and suppressed the expression of mesenchymal markers in HCC cells, along with morphological changes in Huh7 and MHCC97-H cells after incubation with AS-IV, indicating that AS-IV inhibits the EMT of HCC cells.

EMT is a process involved in the conversion of early tumors to aggressive malignancies. Tumor cells undergoing EMT lose their epithelioid phenotype and gain more mesenchymal cell-like features and then intercellular adhesion is reduced and cells acquire invasive ability. Thereby, tumor cells can penetrate the microvascular basic membrane more easily and be transported through the circulation to distant sites $(6,30,31)$. Previous investigations have proven that some constituents of Chinese medicine function as suppressors of EMT (32-34). Our research team also confirmed that SYY inhibited the occurrence of EMT in HCC (20). However, it was not clear which ingredients of SYY played this role. In the present study, we demonstrated that after incubation with AS-IV for $48 \mathrm{~h}$, the expression level of the E-cadherin protein was significantly increased and the mesenchymal markers ( $\mathrm{N}$-cadherin, vimentin, $\alpha$-SMA and Slug) were markedly decreased in both the Huh7 and MHCC97-H cell lines. E-cadherin acts as a tumor suppressor in many types of tumors, since its gene is always inactive in a variety of tumors and activation of the transcription of its gene is sufficient to limit the invasion and metastasis of tumor cells (35-37). The results revealed that AS-IV not only increased the protein level of E-cadherin but also promoted the accumulation of the E-cadherin protein on the cell membrane, thus the cell-cell junctions became more compact and tumor cells were less able to spread. Additionally, AS-IV also downregulated the expression of Slug, which acts as a suppressor of E-cadherin transcription $(38,39)$, thus, AS-IV promoted E-cadherin transcription to some extent.

In the present study, we also revealed the underlying mechanism by which AS-IV suppresses EMT in HCC cells. AKT is commonly regarded as a cancer gene and is overexpressed in many types of solid tumors (40-42). It participates in many basic cellular processes, and it has a close relationship with EMT in cancer (23). The activation of AKT leads to the loss of tumor cell-cell junctions, disruption of tumor cell polarity and morphological changes of tumor cells, enhancing tumor cell motility $(43,44)$. Furthermore, AKT was confirmed to suppress the transcription of the E-cadherin gene by binding to Ets sites and palindromic E-boxes (45). Our results showed that the phosphorylation level of the AKT protein gradually decreased with the increase in the concentration of AS-IV. This was consistent with the inhibition of tumor motility and the increase in the E-cadherin protein. Moreover, the Akt inhibitor GSK690693 was used to further investigate the relationship between AS-IV and AKT. The results from western blotting showed that GSK690693 significantly upregulated the phosphorylation of the AKT protein at the S473 site, as previously described $(27,28)$. However, after treating the cells with AS-IV as well, the phosphorylation of AKT at the S473 site was downregulated. This further illustrated that AS-IV restricted the activity of AKT.

Wnt/ $\beta$-catenin is a critical pathway for EMT. Accumulating evidence suggests that the Wnt/ $\beta$-catenin pathway induces EMT in different tumors $(46,47)$. Blocking Wnt/ $\beta$-catenin-induced EMT suppresses cancer metastasis and progression $(47,48)$. Previous studies demonstrated that $\beta$-catenin forms a complex with E-cadherin on the cell membrane and that downregulation of E-cadherin 
leads to the release of $\beta$-catenin and the translocation of $\beta$-catenin to the nucleus, fully activating the $\mathrm{Wnt} / \beta$-catenin pathway $(25,26)$. Furthermore, $\beta$-catenin is also modulated by GSK-3 $\beta$. It has been proven that GSK-3 $\beta$ induces the phosphorylation of $\beta$-catenin and forces it to undergo ubiquitin/ proteasome-mediated degradation (45). GSK-3 $\beta$ is also a proven target gene of AKT and phosphorylated Akt is known to induce an inactive form of GSK-3 $\beta$ by phosphorylating its Ser9 residues (49). This constitutes an important event in the stabilization of $\beta$-catenin and its subsequent translocation to the nucleus. Our results showed that AS-IV and GSK690693 markedly decreased the phosphorylation of Ser9 residues of GSK-3 $\beta$ respectively, and it was more effective by combining them together, which was consistent with previous studies (49). This indicated that AS-IV relieved the inhibition of GSK-3 $\beta$ by downregulating the phosphorylation of Akt, and may have a regulatory role in the expression and location of $\beta$-catenin. Western blotting was performed to examine the effect of AS-IV on the expression and location of the $\beta$-catenin protein. We found that both $100 \mu \mathrm{g} / \mathrm{ml}$ of AS-IV and $10 \mu \mathrm{M}$ of GSK690693 significantly inhibited $\beta$-catenin expression. Additionally, the accumulation of $\beta$-catenin in the nucleus was also decreased after treatment with $100 \mu \mathrm{g} /$ $\mathrm{ml}$ of AS-IV. In addition, the results from western blotting demonstrated that the treatment of Huh7 and MHCC97-H cells with both AS-IV and GSK690693 at the same time, could inhibit the process of EMT more significantly. These results confirm that Akt/GSK-3 $\beta / \beta$-catenin is an objective pathway by which AS-IV suppresses EMT in HCC cells.

However, this study still has some deficiencies. Our results demonstrated that 10 and $50 \mu \mathrm{g} / \mathrm{ml}$ of AS-IV have little effect on $\beta$-catenin and this may be because the dosage is not enough, or there may exist other signaling pathways that modulate EMT that could be mediated by AS-IV. In addition, our preliminary investigation did not explore the effects of AS-IV on the tumor microenvironment in order to achieve the inhibition of tumor metastasis and invasion.

In conclusion, the present study demonstrated that AS-IV suppressed the invasion and metastasis of HCC cells. In addition, the in vitro assays confirmed that the inhibitory effect of AS-IV on EMT was achieved by targeting the Akt/GSK-3 $\beta / \beta$-catenin signaling pathway. AS-IV, is an important component of SYY and our results highlighted its potential as an adjuvant therapy for the treatment of HCC.

\section{Acknowledgements}

The present study was supported by the National Natural Science Foundation of China (nos. 81272565 and 81172275).

\section{References}

1. Torre LA, Bray F, Siegel RL, Ferlay J, Lortet-Tieulent J and Jemal A: Global cancer statistics, 2012. CA Cancer J Clin 65: 87-108, 2015.

2. Chen W, Zheng R, Baade PD, Zhang S, Zeng H, Bray F, Jemal A, Yu XQ and He J: Cancer statistics in China, 2015. CA Cancer J Clin 66: 115-132, 2016.

3. Zhou XD, Tang ZY, Yang BH, Lin ZY, Ma ZC, Ye SL, Wu ZQ, Fan J, Qin LX and Zheng BH: Experience of 1000 patients who underwent hepatectomy for small hepatocellular carcinoma. Cancer 91: 1479-1486, 2001.
4. Waly Raphael S, Yangde Z and Yuxiang C: Hepatocellular carcinoma: Focus on different aspects of management. ISRN Oncol 2012: 421673-421673, 2012.

5. Chaffer CL and Weinberg RA: A perspective on cancer cell metastasis. Science 331: 1559-1564, 2011.

6. Puisieux A, Brabletz T and Caramel J: Oncogenic roles of EMT-inducing transcription factors. Nat Cell Biol 16: 488-494, 2014.

7. Guerram M, Jiang ZZ, Yousef BA, Hamdi AM, Hassan HM, Yuan ZQ, Luo HW, Zhu X and Zhang LY: The potential utility of acetyltanshinone IIA in the treatment of HER2-overexpressed breast cancer: Induction of cancer cell death by targeting apoptotic and metabolic signaling pathways. Oncotarget 6: 21865-21877, 2015.

8. Lam W, Jiang Z, Guan F, Huang X, Hu R, Wang J, Bussom S, Liu SH, Zhao H, Yen Y, et al: PHY906(KD018), an adjuvant based on a 1800 -year-old Chinese medicine, enhanced the anti-tumor activity of Sorafenib by changing the tumor microenvironment. Sci Rep 5: 9384, 2015

9. Huang XY, Wang L, Huang ZL, Zheng Q, Li QS and Tang ZY: Herbal extract 'Songyou Yin' inhibits tumor growth and prolongs survival in nude mice bearing human hepatocellular carcinoma xenograft with high metastatic potential. J Cancer Res Clin Oncol 135: 1245-1255, 2009.

10. Jia QA, Ren ZG, Bu Y, Wang ZM, Zhang QB, Liang L, Jiang XM, Zhang QB and Tang ZY: Herbal compound 'Songyou Yin' renders hepatocellular carcinoma sensitive to oxaliplatin through inhibition of stemness. Evid Based Complement Alternat Med 2012: 908601, 2012.

11. Li X, Qu L, Dong Y, Han L, Liu E, Fang S, Zhang Y and Wang T: A review of recent research progress on the astragalus genus. Molecules 19: 18850-18880, 2014.

12. Boye A, Wu C, Jiang Y, Wang J, Wu J, Yang X and Yang Y: Compound Astragalus and Salvia miltiorrhiza extracts modulate MAPK-regulated TGF- $\beta /$ Smad signaling in hepatocellular carcinoma by multi-target mechanism. J Ethnopharmacol 169: 219-228, 2015.

13. Wang Y, Auyeung KK, Zhang X and Ko JK: Astragalus saponins modulates colon cancer development by regulating calpain-mediated glucose-regulated protein expression. BMC Complement Altern Med 14: 401-401, 2014.

14. Auyeung KK, Han QB and Ko JK: Astragalus membranaceus: A Review of its protection against inflammation and gastrointestinal cancers. Am J Chin Med 44: 1-22, 2016.

15. Li W and Fitzloff JF: Determination of astragaloside IV in Radix astragali (Astragalus membranaceus var. monghulicus) using high-performance liquid chromatography with evaporative lightscattering detection. J Chromatogr Sci 39: 459-462, 2001.

16. Lai PK, Chan JY, Cheng L, Lau CP, Han SQ, Leung PC, Fung KP and Lau CB: Isolation of anti-inflammatory fractions and compounds from the root of Astragalus membranaceus. Phytother Res 27: 581-587, 2013.

17. Zhao M, Zhao J, He G, Sun X, Huang X and Hao L: Effects of astragaloside IV on action potentials and ionic currents in guinea-pig ventricular myocytes. Biol Pharm Bull 36: 515-521, 2013.

18. Zhang L, Li Z, He W, Xu L, Wang J, Shi J and Sheng M: Effects of astragaloside IV against the TGF- $\beta 1$-induced epithelialto-mesenchymal transition in peritoneal mesothelial cells by promoting Smad 7 expression. Cell Physiol Biochem 37: 43-54, 2015.

19. Qi W, Niu J, Qin Q, Qiao Z and Gu Y: Astragaloside IV attenuates glycated albumin-induced epithelial-to-mesenchymal transition by inhibiting oxidative stress in renal proximal tubular cells. Cell Stress Chaperones 19: 105-114, 2014.

20. Xiong W, Ren ZG, Qiu SJ, Sun HC, Wang L, Liu BB, Li QS, Zhang W, Zhu XD, Liu L, et al: Residual hepatocellular carcinoma after oxaliplatin treatment has increased metastatic potential in a nude mouse model and is attenuated by Songyou Yin. BMC Cancer 10: 219-219, 2010.

21. Tian J, Tang ZY, Ye SL, Liu YK, Lin ZY, Chen J and Xue Q: New human hepatocellular carcinoma (HCC) cell line with highly metastatic potential (MHCC97) and its expressions of the factors associated with metastasis. Br J Cancer 81: 814-821, 1999.

22. Xue G, Restuccia DF, Lan Q, Hynx D, Dirnhofer S, Hess D, Rüegg $\mathrm{C}$ and Hemmings BA: Akt/PKB-mediated phosphorylation of Twist1 promotes tumor metastasis via mediating cross-talk between PI3K/Akt and TGF- $\beta$ signaling axes. Cancer Discov 2: 248-259, 2012. 
23. Hsu CY, Lin CH, Jan YH, Su CY, Yao YC, Cheng HC, Hsu TI, Wang PS, Su WP, Yang CJ, et al: Huntingtin-interacting protein-1 is an early-stage prognostic biomarker of lung adenocarcinoma and suppresses metastasis via Akt-mediated epithelial-mesenchymal transition. Am J Respir Crit Care Med 193: 869-880, 2016.

24. Lamouille S, Xu J and Derynck R: Molecular mechanisms of epithelial-mesenchymal transition. Nat Rev Mol Cell Biol 15: 178-196, 2014

25. Chen HN, Yuan K, Xie N, Wang K, Huang Z, Chen Y, Dou Q, $\mathrm{Wu}$ M, Nice EC, Zhou ZG, et al: PDLIM1 stabilizes the E-cadherin/ $\beta$-catenin complex to prevent epithelial-mesenchymal transition and metastatic potential of colorectal cancer cells. Cancer Res 76: 1122-1134, 2016.

26. Murata-Kamiya N, Kurashima Y, Teishikata Y, Yamahashi Y, Saito Y, Higashi H, Aburatani H, Akiyama T, Peek RM Jr, Azuma T, et al: Helicobacter pylori CagA interacts with E-cadherin and deregulates the $\beta$-catenin signal that promotes intestinal transdifferentiation in gastric epithelial cells Oncogene 26: 4617-4626, 2007.

27. Rhodes N, Heerding DA, Duckett DR, Eberwein DJ, Knick VB, Lansing TJ, McConnell RT, Gilmer TM, Zhang SY, Robell K, et al: Characterization of an Akt kinase inhibitor with potent pharmacodynamic and antitumor activity. Cancer Res 68 : 2366-2374, 2008.

28. Levy DS, Kahana JA and Kumar R: AKT inhibitor, GSK690693, induces growth inhibition and apoptosis in acute lymphoblastic leukemia cell lines. Blood 113: 1723-1729, 2009.

29. Zhang J, Wang P, Ouyang H, Yin J, Liu A, Ma C and Liu L: Targeting cancer-related inflammation: Chinese herbal medicine inhibits epithelial-to-mesenchymal transition in pancreatic cancer. PLoS One 8: e70334, 2013

30. Hanahan D and Weinberg RA: Hallmarks of cancer: The next generation. Cell 144: 646-674, 2011.

31. Qi X, Zhang L and Lu X: New insights into the epithelial-tomesenchymal transition in cancer. Trends Pharmacol Sci 37: 246-248, 2016

32. Lai YJ, Tai CJ, Wang CW, Choong CY, Lee BH, Shi YC and Tai CJ: Anti-cancer activity of Solanum nigrum (AESN) through suppression of mitochondrial function and epithelial-mesenchymal transition (EMT) in breast cancer cells. Molecules 21 : pii: E553, 2016.

33. Lin W, Zhuang Q, Zheng L, Cao Z, Shen A, Li Q, Fu C, Feng J and Peng J: Pien Tze Huang inhibits liver metastasis by targeting TGF- $\beta$ signaling in an orthotopic model of colorectal cancer Oncol Rep 33: 1922-1928, 2015.

34. Lin X, Yi Z, Diao J, Shao M, Zhao L, Cai H, Fan Q, Yao X and Sun X: ShaoYao decoction ameliorates colitis-associated colorectal cancer by downregulating proinflammatory cytokines and promoting epithelial-mesenchymal transition. J Trans Med 12: 105-105, 2014

35. Li Y, Chen CQ, He YL, Cai SR, Yang DJ, He WL, Xu JB and Zan WH: Abnormal expression of E-cadherin in tumor cells is associated with poor prognosis of gastric carcinoma. J Surg Oncol 106: 304-310, 2012.
36. Siu MK, Wong ES, Kong DS, Chan HY, Jiang L, Wong OG, Lam EW, Chan KK, Ngan HY, Le XF, et al: Stem cell transcription factor NANOG controls cell migration and invasion via dysregulation of E-cadherin and FoxJ1 and contributes to adverse clinical outcome in ovarian cancers. Oncogene 32 : 3500-3509, 2013.

37. Qualtrough D, Rees P, Speight B, Williams AC and Paraskeva C: The Hedgehog inhibitor cyclopamine reduces $\beta$-catenin-Tcf transcriptional activity, induces E-cadherin expression, and reduces invasion in colorectal cancer cells. Cancers 7: 1885-1899, 2015.

38. Wang SP, Wang WL, Chang YL, Wu CT, Chao YC, Kao SH, Yuan A, Lin CW, Yang SC, Chan WK, et al: p53 controls cancer cell invasion by inducing the MDM2-mediated degradation of Slug. Nat Cell Biol 11: 694-704, 2009.

39. Fernando RI, Litzinger M, Trono P, Hamilton DH, Schlom J and Palena C: The T-box transcription factor Brachyury promotes epithelial-mesenchymal transition in human tumor cells. J Clin Invest 120: 533-544, 2010 .

40. Lee JK, Phillips JW, Smith BA, Park JW, Stoyanova T, McCaffrey EF, Baertsch R, Sokolov A, Meyerowitz JG, Mathis C, et al: N-Myc drives neuroendocrine prostate cancer initiated from human prostate epithelial cells. Cancer Cell 29: 536-547, 2016.

41. Toker A and Rameh L: PIPPing on AKT1: How many phosphatases does it take to turn off PI3K? Cancer Cell 28: 143-145, 2015.

42. Mayer IA and Arteaga CL: The PI3K/AKT pathway as a target for cancer treatment. Annu Rev Med 67: 11-28, 2016.

43. Attoub S, Arafat K, Hammadi NK, Mester J and Gaben AM: Akt2 knock-down reveals its contribution to human lung cancer cell proliferation, growth, motility, invasion and endothelial cell tube formation. Sci Rep 5: 12759, 2015.

44. Zhang Y, Liu S, Wang L, Wu Y, Hao J, Wang Z, Lu W, Wang XA, Zhang F, Cao Y, et al: A novel PI3K/AKT signaling axis mediates Nectin-4-induced gallbladder cancer cell proliferation, metastasis and tumor growth. Cancer Lett 375: 179-189, 2016.

45. Larue L and Bellacosa A: Epithelial-mesenchymal transition in development and cancer: Role of phosphatidylinositol 3 kinase/AKT pathways. Oncogene 24: 7443-7454, 2005.

46. Gu Y, Wang Q, Guo K, Qin W, Liao W, Wang S, Ding Y and Lin J: TUSC3 promotes colorectal cancer progression and epithelialmesenchymal transition (EMT) through WNT/ $\beta$-catenin and MAPK signalling. J Pathol 239: 60-71, 2016.

47. Lee SC, Kim OH, Lee SK and Kim SJ: IWR-1 inhibits epithelial-mesenchymal transition of colorectal cancer cells through suppressing Wnt/ $\beta$-catenin signaling as well as survivin expression. Oncotarget 6: 27146-27159, 2015.

48. Bernaudo S, Salem M, Qi X, Zhou W, Zhang C, Yang W, Rosman D, Deng Z, Ye G, Yang B, et al: Cyclin G2 inhibits epithelial-to-mesenchymal transition by disrupting Wnt/ $\beta$-catenin signaling. Oncogene 35: 4816-4827, 2016.

49. Soutto M, Peng D, Katsha A, Chen Z, Piazuelo MB, Washington MK, Belkhiri A, Correa P and El-Rifai W: Activation of $\beta$-catenin signalling by TFF1 loss promotes cell proliferation and gastric tumorigenesis. Gut 64: 1028-1039, 2015. 